Türk. Biyo. Mücadele Derg. 2018, 9 (1): 38-47

Orijinal araştırma (Original article)

\title{
Türkiye'nin Bozcaada (Tenedos) Parazitoit Aleiodes Wesmael, 1838 (Hymenoptera: Braconidae: Rogadinae) Türleri $^{1}$
}

\section{Mitat AYDOĞDU²}

\section{Parasitoid species of Aleiodes Wesmael, 1838 (Hymenoptera: Braconidae: Rogadinae) from Bozcaada (Tenedos) Island, Turkey}

\begin{abstract}
Species of the parasitoid wasp Aleiodes Wesmael, 1838 generally lay their eggs in macrolepidopteran larvae (Noctuoidea and Geometroidea, and rarely Arctioidea, Sphingoidea and Papilionoidea). Therefore, these wasps are called koinobiontendoparasitoids due to their life cycles. For this reason, members of this genus are potentially very important biological control agents against pest insects on Bozcaada (Tenedos) which is an island of Turkey located in the north-eastern part of the Aegean Sea. Solitary, endoparasitoid Aleoides species were collected from pastures, vegetable gardens, orchards, trefoil and olive groves at different altitudes in Bozcaada between 2010 and 2014. The specimens were collected by using sweep nets and were prepared according to taxonomic rules and regulations. Ten species belonging to 3 subgenera of Aleiodes were collected and all species are new records for the fauna of Bozcaada.
\end{abstract}

Keywords: Parasitoid, Aleiodes, Bozcaada, Hymenoptera, Pest

Özet: Parazitoit Aleiodes Wesmael, 1838 arıcıkları yumurtalarını genellikle Macrolepidoptera larvaları (Noctuoidea and Geometroidea, nadiren Arctioidea, Sphingoidea and Papilionoidea) içine bırakırlar. Bundan dolayı, bu arıcıkların yaşam döngüleri koinobiont-endoparasitoit olarak isimlendirilir. $\mathrm{Bu}$ nedenle, bu cinse ait parazitoitler potansiyel olarak zararlı böceklere karşı önemli biyolojik mücadele etmenleridir. Bozcaada (Tenedos), Ege Denizi'nin kuzey doğusundaki Türkiye'nin bir adasıdır. İdari olarak, ada Çanakkale ilinin Bozcaada ilçesini teşkil eder ve Gökçeada (Imbros) ve Marmara Adası'ndan sonra üçüncü büyük Türk adasıdır. Soliter-endoparasitoit Aleiodes türleri 2010-2014 yılları arasında Bozcaada'nın otlak alanları, sebze ve meyve bahçeleri, Yoncalık ve Zeytinlik gibi farklı yüksekliklerinden süpürme atrapları kullanılarak toplandı ve taksonomik kurallara uygun olarak prepare edildi. Aleiodes cinsinden 3 altcinse ait 10 tür tanımlandı ve bütün türler Bozcaada faunası için ilk kayıttır.

\footnotetext{
${ }^{1} \mathrm{Bu}$ çalışma, 16-18 Mayıs 2017 tarihinde Tekirdağ'da düzenlenen AgriBalkan Kongresi'nde poster olarak sunulmuş ve özet olarak basılmıștır.

2 Trakya Üniversitesi, Fen Fakültesi, Biyoloji Bölümü - 22030 Edirne

Sorumlu yazar (Corresponding author) e-mail: maydogdu75@ hotmail.com

Alınış (Received): 15.01.2018 Kabul ediliş (Accepted): 21.03.2018
} 
Türk. Biyo. Mücadele Derg.

Aydoğdu 2018, 9 (1):38-47

Anahtar kelimeler: Parasitoit, Aleiodes, Bozcaada, Hymenoptera, Zararlı

\section{Giriş}

Rogadinae altfamilyası birinci ve ikinci metasomal tergitleri arasındaki derin oluk mevcudiyeti ve birinci tergitin posteroventral kenarı ile ikinci tergitin anteroventral kenarının belirgin olması sayesinde diğer cyclostom brakonidlerden ayrılırlar. (Tobias 1986). Aleiodes cinsi ise dünya çapında en yaygın Rogadinae cinsi olup tür zenginliği ile ilgili tahminler son yıllarda önemli ölçüde değişmiştir. Günümüzde Aleiodes türleri toplam Rogadinae famiyasına ait türlerin yaklaşık yarısını oluşturmaktadır (Aydogdu 2015; Tobias 1986).

Aleiodes Wesmael türleri oldukça büyük bir altfamilya olup, koinobiontendoparazitoit olarak gelişimlerini özellikle makrolepidopterler üzerinde tamamlarlar (Noctuoidea ve Geometroidea, daha az siklıkla da Arctioidea, Sphingoidea ve Papilionoidea). Konukçu kelebek larvası parazitlendikten sonra geçici bir süre tam olarak paralize olmaz ve gelişimine devam eder. Rogadini tribusuna özgü bu türde parazitizm oldukça farklı ve dikkat çekicidir. Parazitoit ve konukçu biyolojisi çalışmalarında mumyalanmış tırtıl kalıntıları önemli tanısal değere sahiptir. (Quicke \& Shaw 2005; Shimbori \& Shaw 2014).

III. jeolojik zamanın sonunda, yaklaşık 2-2.5 milyon yıl önce yer kabuğunun kırılıp çökmesiyle oluşmuş bir deniz olan Ege'nin kuzeydoğu kısmında yer alan Gökçeada ve Bozcaada Anadolu ve Trakya'nın devamı niteliğindedir. Bozcaada konum olarak Gökçeada'nın güneyinde, Çanakkale boğazının Ege çıkısında, Odun iskelesine yaklaşık $6 \mathrm{~km}$. uzaklıkta yer alan ve $36.03 \mathrm{~km}^{2}$ 'lik yüzölçümüyle Gökçeada ve Marmara adalarından sonra Türkiye'nin üçüncü büyük adasıdır. Akdeniz ve karasal iklimin etkisi altında ve hakim bitki örtüsü makiler olan her iki ada flora ve vejetasyon bakımından Batı Anadolu'yu andırmaktadır (Özkan 1999).

Türkiye Rogadinae familyasına ait Aleiodes cinsi türlerinin yayılışları ve habitat tercihleri üzerine yürütülmüş birçok çalışmaya literatürde rastlamak mümkün olmasına rağmen, türlerin yayılışları ve habitat tercihleri hakkındaki bilgiler yeterli değildir. Türkiye'de yalnızca Marmara, Ege ve Kuzeydoğu Anadolu Bölgeleri'nden kayıt bulunmaktadır. (Shenefelt 1975; Fahringer 1922; Beyarslan et al. 2002; Aydoğdu \& Beyarslan 2005, 2006; Öncüer 1991; Aydoğdu 2015; Beyarslan 2015). Bu bağlamda bu çalışmada, Bozcaada faunası Aleiodes türlerini taksonomik yönden değerlendirmek ve Türkiye faunasına tür çeşitliliği açısından katkıda bulunmak amaçlanmıştır.

\section{Materyal ve Yöntem}

Bozcaada'nın Aleiodes faunasının saptanmasında kullanılan araştırma materyali 2010-2014 yılları arasında, Rogadinae altfamilyası ergin bireylerinin aktif olduğu Mayıs-Eylül periyotlarında habitat özellikleri farklı olan alanlardan toplanmıştır. 
Türkiye'nin Bozcaada (Tenedos) Parazitoit AleiodesTürleri

Gündüz saptanan türler ince naylon perdelik tülden yapılmıș atrap yardımıyla meyve ve sebze bahçeleri, zeytinlik, yoncalık ve otsu bitkiler üzerinden toplanmıştır. Ergin bireyler diğer materyalden emgi tüpü yardımı ile ayrılmış, potasyum siyanür $(\mathrm{KCN})$ içeren öldürme şişelerinde öldürülmüş ve depolama kapları içinde ya da gliserinli alkol tüplerine konularak laboratuara getirilmiştir.

Örnekler nemlendirme kaplarında nemlendirilerek preparasyonları yapılmıştır. Materyal, tanıda önem taşıyan morfolojik özellikleri görülebilecek şekilde düzeltilerek, böcek iğnelerine takılmış üçgen kartonlara yapıştırılmıştır. Tanı anahtarında belirtilen vücut kısımlarının ölçümlerinin doğru bir şekilde yapılabilmesi ve karakterlerin en iyi şekilde görülebilmesi için, yapıştırma işlemi örneklerin mesosoma kısmından yapılmıştır. Koleksiyon tekniğine göre etiketlenen örnekler müze materyali haline getirilmiştir (Aydoğdu 2006).

Materyalin taksonomik olarak incelenmesinde Rogadinae altfamilyasına ait Papp (1985), Tobias (1986) ve Shaw et al. (2006) tarafından hazırlanan teşhis anahtarlarından yararlanılmıştır.

\section{Bulgular ve Tartışma}

\section{Altfamilya: Rogadinae Förster, 1862}

\section{Cins: Aleiodes Wesmael, 1838}

\section{Altcins: Aleiodes s. str. Wesmael, 1838}

\section{Aleiodes (Aleiodes) bicolor (Spinola, 1808)}

Toplanan Materyal: Çanakkale-Bozcaada-Amerikan çeşme mevkii, 30. 08. 2012, otsu bitkiler, 80 m., ㅇ, ô; -Bozcaada-Ayazma-Sulubahçe, 03.09.2014, üzüm bağ1 ve sebze bahçesi, 15 m., .+ -Bozcaada-Göztepe, 30.06.2011, otsu bitkiler, meyve bahçesi, 70 m., ; ; -Bozcaada-İğdelik, 08.07 2012, üzüm bağ1 ve otsu

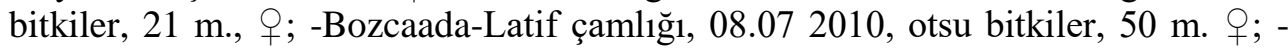
Bozcaada-Merkez, 09.07.2012, yoncalık, otsu bitkiler, 10m., 2우; BozcaadaTuzburnu, 08.07.2012, zeytinlik,otsu bitkiler, 30 m., 1 ․

Türkiye Dağılımı: Afyon, Adapazarı, Ardahan, Aydın, Balıkesir, Bilecik, Bursa, Çanakkale, Denizli, Edirne, Erzurum, İstanbul, Iğdır, İzmit, İzmir, Kars, Kırklareli, Kütahya, Muğla, Tekirdağ, Uşak, Yalova.

Zoocoğrafik Dağılım: Palaearktik.

Konukçu: Lepidoptera, Crambidae: Loxostege sticticalis (Linnaeus 1761); Pyrausta purpuralis (Linnaeus 1758); P. sanguinalis (Linnaeus 1767). Geometridae: Apocheima cinerarius (Erschoff 1874); Archiearis parthenias (Linnaeus 1761); Eupithecia linariata (Denis \& Schiffermüller 1775);Operophtera 
(Hubner 1825); Lycaenidae: Aricia agestis (Denis \& Schiffermüller 1775); A. artaxerxes (Fabricus 1793); Cupido alcetas (Hoffmannsegg 1804); C. minimus (Fuessly 1775); Lysvera coridon (Poda 1761); Plebejus idas (Linnaeus 1761); Polyommatus albicans (Gerhard 1851); P. damon (Denis \& Schiffermüller 1775); P. eros (Ochsenheimer 1808); P. icarus (Rottemburg 1775). Lymantriidae: Dasychira albodentata Bremer 1864; Leucoma salicis (Linnaeus 1758); Noctuidae: Apamea sordens (Hufnagel 1766). Nymphalidae: Maniolajurtina (Linnaeus 1758); Aglais urticae (Linnaeus 1758). Pterophoridae: Emmelina monodactyla (Linnaeus 1758); Hellinsia tephradactyla (Hubner 1813); Zygaenidae: Jordanita chloros (Hubner 1813); J. graeca (Jordan 1907); Rhagades pruni (Denis \& Schiffermüller 1775); Zygaena filipendulae (Linnaeus 1758); Z. lonicerae (Scheven 1777); Z. purpuralis (Brunnich 1763); Z. viciae (Denis \& Schiffermüller 1775).

\section{Aleiodes (Aleiodes) circumscriptus (Nees 1834)}

Toplanan Materyal: Çanakkale-Bozcaada-Ayazma-Sulubahçe, 03.09.2014, üzüm bağ1 ve otsu bitkiler, 15 m., 2 2 $ᄋ$, ô; -Bozcaada-Latif çamlığı, 08.07 2010, otsu bitkiler, $50 \mathrm{~m} .2$. 9 .

Türkiye Dağılımı: Afyon, Aydın, Adapazarı, Ardahan, Artvin, Bursa, Edirne, Çanakkale, Erzurum, Iğdır, İzmir, Kütahya, Manisa, Muğla, Kars, Muş, Tekirdağ.

Zoocoğrafik Dağılım: Palaearktik.

Konukçu: Lepidoptera, Arctiidae: Atolmis rubricollis (Linnaeus 1758); Lymantriidae: Leucoma alicis (Linnaeus 1758); Gelechiidae: Caryocolum amaurella(Hering 1924). Geometridae: Thera variata (Denis \& Schiffermüller 1775); Abraxas grossulariata Linnaeus 1758; Bupalus piniarius Linnaeus 1758; Cyclophora ruficiliaria (Herrich-Schaffer 1855); Epirrhoe galiatav (Denis \& Schiffermüller 1775); Epirrita autumnata (Borkhausen 1794); Eupithecia alliaria (Staudinger 1870); E. linariata (Denis \& Schiffermüller 1775); E. subfuscata (Haworth 1809); Gymnoscelis rufifasciata (Haworth 1809); Hylaea fasciaria (Linnaeus 1758); Lycia isabellae (Harrison 1914); Noctuidae: Apameacrenata (Hufnagel 1766); Autographa gamma (Linnaeus 1758); Diarsia rubi (Vieweg 1790); Helicoverpa armigera (Hubner 1808); Hoplodrina octogenaria (Goeze 1781); Lithomoia solidaginis (Hubner 1803); Mamestra brassicae (Linnaeus 1758); Noctua fimbriata (Schreber 1759); N. pronuba (Linnaeus 1758); Orthosia gracilis (Denis \& Schiffermüller 1775); Orthosia incerta (Hufnagel 1766); O. stabilis (Denis \& Schiffermüller 1775); Penicillaria jocosatrix Guenee 1852; Pseudaletia unipuncta (Haworth 1809); Spodoptera exigua (Hubner 1808); Xestia agathina (Duponchel 1827); X. baja (Denis \& Schiffermüller 1775); X. xanthographa (Denis \& Schiffermüller 1775).Pterophoridae: Pterophorus pentadactyla (Linnaeus 1758); Pyralidae: Dioryctria abietella (Denis \& Schiffermüller 1775); Ebulea crocealis (Denis \& Schiffermüller 1775); Tortricidae: Archips rosana (Linnaeus 1758); Croesia bergmanniana Linnaeus 
Türkiye'nin Bozcaada (Tenedos) Parazitoit AleiodesTürleri

1758; C. holmiana Linnaeus 1758; Cydia conicolana (Heylaerts 1874); C. strobilella (Linnaeus 1758); Pammene amygdalana (Duponchel 1842); P. gallicolana (Lienig \& Zeller 1846); Tortrix viridana (Linnaeus 1758); Zeiraphera griseana (Hubner 1799).

\section{Aleiodes (Aleiodes) gastritor (Thunberg, 1822)}

Toplanan Materyal: Çanakkale-Bozcaada-Merkez, 09.07.2012, yoncalık, otsu bitkiler, 10m., 2 +,$+ O^{\top}$.

Türkiye Dağılımı: Ardahan, Balıkesir, Çanakkale, Edirne, Erzurum, İzmir, Kars, Kırklareli, Muğla.

Genel Dağılımı: Holoarktik, Oseanik, Oryantal.

Konukçu: Lepidoptera, Crambidae: Ostrinia nubilalis (Hubner 1796); Phlyctaenia coronata (Hufnagel 1767). Drepanidae: Cilix glaucata (Scopoli 1763); Elachistidae: Depressaria absynthiella (Herrich-Schaffer 1865). Geometridae: Alsophila pometaria (Harris 1841); Apocheima cinerarius (Erschoff 1874); A. hispidaria (Denis \& Schiffermüller 1775); Chiasmia clathrata (Linnaeus 1758); Chloroclystis v-ata (Haworth 1809); Digrammia gnophosaria (Guenee 1857); Epirrita autumnata (Borkhausen 1794); Erannis defoliaria (Clerck 1759); Eupithecia alliaria (Staudinger 1870); E. miserulata (Grote 1863); E. pusillata (Denis \& Schiffermüller 1775); Glena cribrataria (Guenee 1858); Hylaea fasciaria (Linnaeus 1758); Hypagyrtis unipunctata (Haworth 1809); Isturgia limbaria (Fabricius 1775); Lycia hirtaria (Clerck 1760); Lycia pomonaria (Hubner 1790); Operophtera brumata (Linnaeus 1758); Phthonveria atrilineata (Butler 1881); Tephrina arenacearia (Denis \& Schiffermüller 1775); Lasiocampidae: Malacosoma neustria (Linnaeus 1758); Lymantriidae: Euproctis chrysorrhoea (Linnaeus 1758); E. similis (Fuessly 1775); Hypena scabra (Fabricius 1798); Leucoma salicis (Linnaeus 1758). Noctuidae: Agrapha agnata (Staudinger 1892); Autographa gamma (Linnaeus 1758); Harpyia hermelina (Stephens 1829); Helicoverpa armigera (Hubner 1808); Pseudaletia unipuncta (Haworth 1809); Spodoptera exigua (Hubner 1808); Trichoplusia ni (Hubner 1803); Notodontidae: Cerura vinula (Linnaeus 1758); Thaumetopoea processionea (Linnaeus 1758). Yponomeutidae: Prays oleae (Bernard 1788); Tortricidae: Archips rosana (Linnaeus 1758); Lobesia botrana (Denis \& Schiffermüller 1775).

\section{Aleiodes (Aleiodes) nocturnus (Telenga 1941)}

Toplanan Materyal: Çanakkale-Bozcaada-Göztepe, 30.06.2011, otsu bitkiler, meyve bahçesi, $70 \mathrm{~m}$., q.

Türkiye Dağılımı: Balıkesir, Çanakkale, Edirne, Erzurum, İzmir, Kırklareli, Manisa, Uşak. 
Zoocoğrafik Dağılım: Palaearktik.

Konukçu: Lepidoptera, Lymantriidae: Euproctis karghalica (Moore 1878); Noctuidae: Helicoverpa armigera (Hubner 1808); H. zea (Boddie 1850). Notodontidae: Cerura menciana (Moore 1877); C. vinula (Linnaeus 1758); Harpyia hermelina (Goeze 1781).

\section{Aleiodes (Aleiodes) pallidator (Thunberg 1822)}

Toplanan Materyal: Çanakkale-Bozcaada-Tuzburnu, 08.07.2012, otsu bitkiler, $30 \mathrm{~m} ., 1$.

Türkiye Dağılımı: Ardahan, Balıkesir, Edirne, Erzurum, İzmir, Kars, Kütahya, Muğla, Tekirdăg.

Zoocoğrafik Dağılım: Holoarktik, Neotropikal, Oriental.

Konukçu: Lepidoptera, Gelechiidae: Anarsia lineatella (Zeller 1839); Caryocolum amaurella (Hering 1924); Geometridae: Bupalus piniarius (Linnaeus 1758); Cyclophora pendularia (Hufnagel 1767); C. punctaria (Linnaeus 1758); C. quercimontaria (Bastelberger 1897); Dyscia conspersaria (Denis \& Schiffermüller 1775); Eupithecia pimpinellata (Hubner 1813); Hylaea fasciaria (Linnaeus 1758); Lasiocampidae: Selenephera lobulina (Schiffermüller 1775). Lymantriidae: Euproctis karghalica (Moore 1878); Orgyia definita (Packard 1865); O. leucostigma (Smith 1797). Noctuidae: Agrotis segetum (Denis \& Schiffermüller 1775); Heliothis viriplaca (Hufnagel 1766); Helicoverpa armigera (Hubner 1808); H. zea (Boddie 1850); Spodoptera exigua (Hubner 1808); Notodontidae: Cerura menciana (Moore 1877); C. vinula (Linnaeus 1758); Harpyia hermelina (Goeze 1781); Psychidae: Megalophanes viciella (Denis \& Schiffermüller 1775). Tortricidae: Apotomis sororculana (Zetterstedt 1839).

\section{Aleiodes (Aleiodes) signatus (Nees 1811)}

Toplanan Materyal: Çanakkale-Bozcaada-Hacıhasan tepe, 07.06 2010, otsu bitkiler, $45 \mathrm{~m}$. . .

Türkiye Dağılımı: Ardahan, Afyon, Bilecik, Bursa, Edirne, Erzurum, Iğdır, İstanbul, İzmit, Kars, Kırklareli, Tekirdağ.

Zoocoğrafik Dağılım: Palaearktik.

Konukçu: Lepidoptera, Arctiidae: Arctia caja (Linnaeus 1758); Coscinia cribraria (Linnaeus 1758); Epicallia villica (Linnaeus 1758); Ocnogyna baetica (Rambur 1837). Lymantriidae: Euproctis chrysorrhoea (Linnaeus 1758); E. similis (Fuessly 1775); Gynaephora selenitica (Esper 1789); Orgyia antiqua (Linnaeus 1758); O. aurolimbata (Guenee1835). Lasiocampidae: Euthrix potatoria (Linnaeus 1758). Noctuidae: Acronicta psi (Linnaeus 1758); A. rumicis (Linnaeus 1758); 
Türkiye'nin Bozcaada (Tenedos) Parazitoit AleiodesTürleri

Autographa gamma (Linnaeus 1758); Noctua fimbriata (Schreber 1759); N. pronuba (Linnaeus 1758). Notodontidae: Taumetopoea pityocampa (Denis \& Schiffermüller 1775); T. processionea (Linnaeus 1758). Tortricidae: Spilonota ocellana (Denis \& Schiffermüller 1775).

Altcins: Chelonorhogas Enderlein 1912

Aleiodes (Chelonorhogas) apicalis (Brullé 1832)

Toplanan Materyal: Çanakkale-Bozcaada-Tuzburnu, 08.07.2012, otsu bitkiler, 30 m., 1 ․

Türkiye Dağılımı: Adapazarı, Afyon, Balıkesir, Bursa, Bilecik, Çanakkale, Denizli, Edirne, Kurklareli, Tekirdağ.

Zoocoğrafik Dağılım: Batı Palaearktik.

Konukçu: Lepidoptera, Lasiocampidae: Philudoria pototoria (Linnaeus 1758); Mamestra brassicae (Linnaeus 1758); Noctuidae: Autographa gamma (Linnaeus 1758); Discestra trifolii (Hufnagel 1766).

Aleiodes (Chelonorhogas) rufipes (Thomson 1892)

Toplanan Materyal: Çanakkale-Bozcaada-Hacıhasan tepe, 07.06 2010, otsu bitkiler, $45 \mathrm{~m}$. . .

Türkiye Dă̆ılımı: Çanakkale, Muğla, Tekirdağ.

Zoocoğrafik Dağılım: Palaearktik.

Konukçu: Bilinmemektedir.

Altcins: Neorhogas Szépligeti 1906

Aleiodes (Neorhogas) dissector (Nees 1834)

Toplanan Materyal: Çanakkale-Bozcaada-Merkez-Seramit tepe, 30.05.2013, zeytinlik, üzüm bahçesi, otsu bitkiler, 50 m., 1 ․

Türkiye Dağılımı: Ardahan, Denizli.

Zoocoğrafik Dağılım: Palaearktik.

Konukçu: Lepidoptera, Noctuidae: Acronicta rumicis (Linnaeus 1758); A. tridens (Denis \& Schiffermüller, 1775); Orthosia incerta (Hufnagel 1766).

Aleiodes (Neorhogas) ductor (Thunberg 1822)

Toplanan Materyal: Çanakkale-Bozcaada-Tuzburnu, 08.07.2012, zeytinlik,otsu bitkiler, 30 m., 1 ㅇ. 
Türkiye Dağılımı: Afyon, Ardahan, Aydın, Çanakkale, Denizli, Edirne, Erzurum, Iğdır, İzmir, Kırklareli, Kütahya, Manisa, Muş, Tekirdağ.

Zoocoğrafik Dağılım: Palaearktik.

Konukçu: Lepidoptera, Lasiocampidae: Anarta (Calocestra) trifolii (Hufnagel 1766); Autographa gamma (Linnaeus 1758); Euthrix potatoria (Linnaeus 1758); Mamestra brassicae (Linnaeus 1758); Nymphalidae: Brenthis ino (Rottemburg 1775); Sesiidae: Synanthedon scoliaeformis (Borkhausen 1789).

Marmara Bölgesi'nin Bozcaada Rogadinae faunasını saptamak amacıyla çeşitli lokalite ve habitatlarda yapılan araştırmalar sonucunda Rogadinae altfamilyasına ait Aleiodes altcinsi (6 tür), Chelonorhogas altcinsi (2 tür) ve Neorhogas (2 tür) olmak üzere toplam 10 tür saptanmıştır. Saptanan yararlı türlerin hepsi Bozcaada Rogadinae faunası için yeni kayıttır.

Özellikle alacakaranlıkta aktif olan ergin Rogadinae altfamilyası üyeleri farklı habitatlarda yaygınlık göstermektedir. Bu çalışma sonucunda toplanan örneklerin dağılımının özellikle otsu bitkiler ve meyve-sebze bahçelerinde yoğunlaştığı gözlenmiştir. Rogadinae familyası içinde de ekolojik valansı yüksek bir tür olan A.(A.) bicolor araştırma sahasında özellikle otsu bitkilerin yoğun olduğu habitatlardan tespit edilmiştir. Türkiye'nin diğer bölgelerinde olduğu gibi çalışmanın yürütüldüğü alanda da tarımsal zararlılardan korunmak için kimyasal ajanların kullanımının yoğun olmasından dolayı biyolojik mücadele için önem arz eden yararlı Rogadinae altfamilyasına ait türlerin sayısında da önemli bir azalma olduğu düşünülmektedir.

Tarımsal alanlarda zararlılara karşı yoğun ve bilinçsiz tarımsal ilaç kullanımı çevre ve insan sağlığını olumsuz yönde etkilemektedir. Doğal dengenin bozulması özellikle biyolojik mücadelede kullanılabilecek yararlı türlerin ortadan kalkması ve sekonder zararlıların salgın riski yanlış uygulanan tarımsal ilaçlamalardan kaynaklanabilmektedir. Doğal denge bozulduğunda, ekosistem dengesi bozulur ve geri dönüşümü olmayan ekolojik problemler ortaya çıkabilir.

Dünyadaki tüm gelişmiş ülkeler tüm alanlardaki araştırmalara paralel olarak bir taraftan fauna araştırmalarını, diğer taraftan biyolojik mücadele uygulamalarını hızla yürütmektedirler. Biyolojik Mücadele uygulamalarının artması etkin olarak kullanılabilecek faydalı tür sayısına da bağlıdır. Bu nedenle biyolojik mücadele etmenlerine yönelik taksonomik çalışmalar son derece önemlidir. Gerçekleştiren bu çalışmada bu yönüyle değerlendirildiğinde ülkemiz faydalı etmen faunasına katkı sağlamaktadır. Bulunan bu faydalı etmenlerle ilgili uygun biyoloji çalışmalarının yapılması ve devamında da biyolojik mücadele uygulamalarında yer almaları taksonomik çalışmaların başarısını daha da arttıracaktır. 


\section{Kaynaklar}

Aydogdu M. 2015. Ege Bölgesi Aleiodes Wesmael, 1838 (Hymenoptera: Braconidae: Rogadinae) faunasına katkılar. Anadolu Doğa Bilimleri Dergisi, 6(2): 126-133.

Aydogdu M. 2016. Doğu Anadolu Bölgesi Cheloninae Förster, 1862 (Hymenoptera, Braconidae) faunası üzerine taksonomik bir araştırma. Türkiye Biyolojik Mücadele Dergisi,7(2): 123-138.

Aydogdu M. \& A. Beyarslan 2005. The first records of Aleiodes Wesmael 1838 (Hymenoptera: Braconidae: Rogadinae) the fauna of Thrace region of Turkey. Linzer Biologische Beitrage, 37: 185-193.

Aydogdu M. \& A. Beyarslan 2006. First records of Aleiodes Wesmael, 1838 species in East Marmara Region of Turkey (Hymenoptera: Braconidae: Rogadinae). Acta Entomologica Slovenica, 14: 81-88.

Beyarslan A. 2015.Taxonomic survey on the Rogadinae Förster, 1862 (Hymenoptera, Braconidae) in the northeastern Anatolian region, Turkey. Turkish Journal of Zoology, 39(4):1-9.

Beyarslan A., F. İnanc, Ö. Cetin, \& M. Aydoğdu 2002. Braconidae species of the Turkish Aegean region. In: Melika G, Thuroczy C, editors. Parasitic Wasps: Evolution, Systematics, Biodiversityve Biological Control. International Symposium: Parasitic Hymenoptera: Taxonomy ve Biological Control; 14-17 May2001; Kszeg, Hungary. Budapest: Agroinform, pp. 285-290.

Fahringer J. 1922. Hymenopterologische Ergebnisse einer Studienreise nach der Turkei und Kleinasien. Archives du muséum d'histoire Natürelle, 88: 149-222.

Quicke D.L.J. \& M.R. Shaw 2005. First host records for the rogadine genera Rogasodes Chen ve He veCanilirogas van Achterberg ve Chen (Hymenoptera: Braconidae) with description of a new species ve survey of mummy types within Rogadinae s. str. Journal of Natural History, 39: 3525-3542.

Papp J. 1985. Contributions to the Braconid fauna of Hungary, VII. Rogadinae (Hym.: Braconidae). Folia Entomologica Hungarica, 46: 143-164.

Shaw M.S., P.M. Marsh \& J.C. Fortier 2006. Revision of North American Aleiodes Wesmael (Part 8): the coxalis Spinola species-group (Hymenoptera: Braconidae: Rogadinae). Zootaxa, 1314: 1-30.

Shenefelt R.D. 1975. Braconidae 8: Exothecinae, Rogadinae. In: van der Vecht J, Shenefelt RD, editors. Hymenopterorum Catalogus (novo editio). The Hague, Netherlves: W. Junk B.V., 1115- 262 pp.

Shimbori E.M. \& S.R. Shaw 2014. Twenty-four new species of Aleiodes Wesmael from the eastern Vees of Ecuador with associated biological information (Hymenoptera, Braconidae, Rogadinae). ZooKeys, 405: 1-81.

Tobias V.I. 1986. [Order Hymenoptera. Family Braconidae]. In: Medvedev G. S. (ed.), 'Opredelitel Nasekomych Evrospeiskoi Tsasti SSSR 3, Peredpontdatokrylye 4. Opr. Faune SSSR.' 145: 1-501. [Keys to the insects of the European part of USSR. Hymenoptera].

Yu D.S., C. van Achterberg \& K. Horstmann 2012. World Ichneumonoidea 2004. taxonomy, biology, morphology and distribution (Braconidae). Taxapad 2005 (Scientific Names for Information Management) Interactive Catalogue on DVD/ CDROM. Vancouver, Canada. 
Öncüer C.1991. Türkiye bitki zararlısı böceklerinin parazit ve predatör katalogu. Izmir Ege Üniversitesi Ziraat Fakültesi, Ofset Basımevi, 505: 180-197.

Özkan M. 1999. Gökçeada ve Bozcaada Kemirici faunası (Mammalia; Rodentia). Turkish Journal of Zoology, 23(1):133-147. 